\title{
Chronic myeloid leukaemia occurring in a patient with hairy cell leukaemia
}

\author{
F A Wandroo, D Bareford, F El-Jehani
}

\begin{abstract}
Occurrences of second malignancies in hairy cell leukaemia are well recognised. Most of these malignancies are either solid tumours or lymphoproliferative disorders. The association of myeloproliferative disorders with hairy cell leukaemia (HCL) is very rare. This report describes a case of a patient with HCL who after remaining in remission developed Philadelphia chromosome positive chronic myeloid leukaemia (CML), which rapidly transformed to acute lymphoblastic leukaemia with further cytogenetic abnormalities.

(f Clin Pathol 2000;53:940-941)
\end{abstract}

Keywords: hairy cell leukaemia; chronic myeloid leukaemia; acute lymphoblastic leukaemia

Hairy cell leukaemia (HCL) is a well recognised lymphoproliferative disorder. This condition is characterised by splenomegaly, pancytopenia, and typical hairy cells in the peripheral blood and bone marrow. Lymphadenopathy is rare. The white blood cell count rarely goes above $20 \times 10^{9} /$ litre but neutropenia and monocytopenia are constant features. ${ }^{1}$ The diagnosis is confirmed by bone marrow biopsy and immunophenotyping. Tartarate resistant acid phosphatase (TRAP) demonstrated on hairy cells is quite specific for hairy cell leukaemia. ${ }^{2}$ The incidence of second malignancies in hairy cell leukaemia is reported as $8.7 \% .^{3}$ Most of the second malignancies occurring in hairy cell leukaemia are either solid tumours or other lymphoproliferative disorders. ${ }^{4}$ The occurrence of myeloproliferative disorders in hairy cell leukaemia is extremely rare. There has been only one case of Philadelphia chromosome positive chronic myelogenous leukaemia with hairy cell leukaemia reported in the literature. ${ }^{2}$ We report another such case of a patient with hairy cell leukaemia who after remaining in remission for four years developed Philadelphia chromosome positive chronic myeloid leukaemia that rapidly transformed to acute lymphoblastic leukaemia.

\section{Case report}

A 45 year old man presented to his general practitioner with facial cellulitis and recurrent chest infections. He also had a history of weight loss and night sweats for the previous two years. Physical examination revealed an $8 \mathrm{~cm}$ splenomegaly. Blood counts revealed: haemoglobin, $129 \mathrm{~g} /$ litre; white cell count, $1.8 \times 10^{9} /$ litre (differential: $32 \%$ neutrophils, $60 \%$ lymphocytes, and an absolute monocytopenia); and platelet count, $58 \times 10^{9} /$ litre. A peripheral blood smear revealed normal red blood cell morphology but a lymphocyte population showing typical morphological features of hairy cells; that is, abundant granular cytoplasm, irregular cytoplasmic projections, oval to slightly indented nuclei containing dispersed chromatin, and inconspicuous nucleoli. A bone marrow aspirate was aparticulate but cellular and revealed $20-25 \%$ hairy cells, which were positive for CD19, CD20, CD22, CD25, CD11c, and surface $\kappa$ chains and negative for CD 5 by surface marker analysis; $10 \%$ of these cells were positive for TRAP. A trephine biopsy was hypercellular, showing almost complete replacement of normal haematopoietic tissue by a diffuse infiltrate of moderate sized lymphocytes with the typical lacunae appearance of hairy cells (fig 1). The patient was treated with weekly deoxycoformycin $(8 \mathrm{mg}$ intravenously) initially for four weeks, then fortnightly for five months. He went into remission with complete resolution of splenomegaly and a bone marrow showing no infiltrate. He remained well and in remission for four years, at which time his white blood cell count suddenly increased to $20 \times 10^{9} /$ litre, with a differential revealing $56 \%$ neutrophils, $6 \%$ lymphocytes, $1.3 \%$ basophils, $15 \%$ metamyelocytes, $2 \%$ myelocytes, $0.9 \%$ monocytes, and $0.2 \%$ eosinophils. His bone marrow was hypercellular showing increased granulopoiesis, with a pronounced left shift revealing $23 \%$ blasts, $2 \%$ promyelocytes, $10 \%$ myelocytes, $12 \%$ metamyelocytes, and 33\% neutrophils. A trephine biopsy showed areas of increased granulopoiesis with an increase in immature forms and some lacunae of lymphocytes. Cytogenetics confirmed a $t(9 ; 22)$ translocation and a diagnosis of chronic myeloid leukemia (CML) was made. The patient was tissue typed with his siblings and started on interferon. $\mathrm{He}$ showed a good initial response with his white blood cell count falling to $4 \times 10^{9} /$ litre. However, after a couple of weeks his white blood cell count increased again to $20 \times 10^{9} /$ litre, with the blood film showing $85 \%$ lymphoblasts. Cytogenetics revealed additional chromosomal abnormalities (loss of the short arm and a part of the long arm of chromosome 9) in addition to the $t(9 ; 22)$ translocation, indicating clonal evolution consistent with disease progression and transformation. Cytochemistry and immunophenotyping confirmed CALLA+, precursor B cell acute lymphoblastic leukaemia (ALL; 76\% CD10+ cells, 97\% CD19+ cells). The trephine biopsy revealed the complete replacement of normal haemopoiesis by monomorphic blast cells. The patient was started on the 


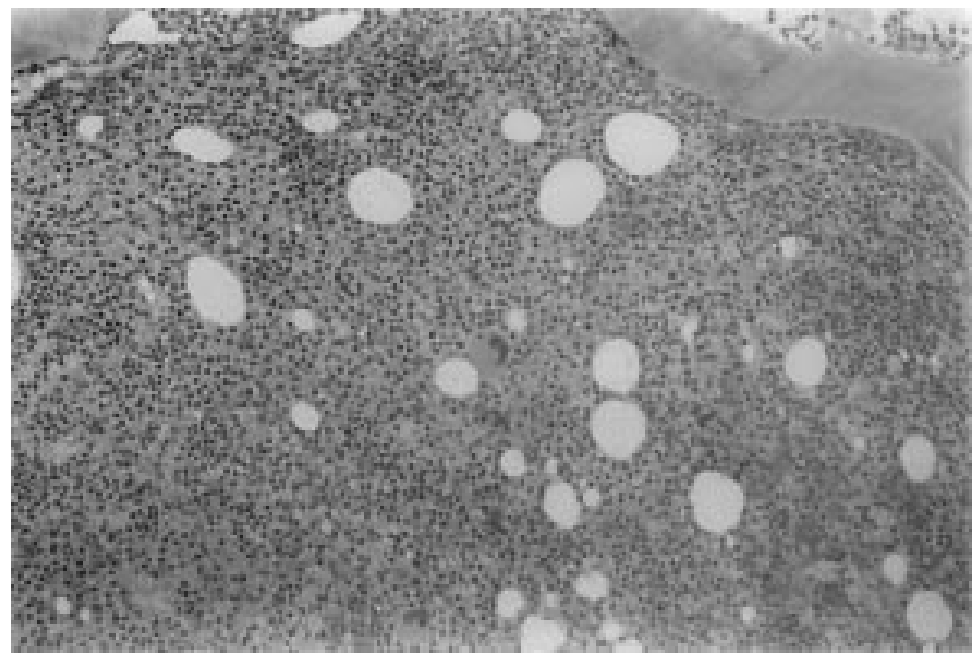

Figure 1 Iliac crest biopsy, showing diffuse infiltration by hairy cells with their abundant cytoplasm and wide cell to cell distances.

UKALL XII protocol. Unfortunately, in the third week of remission induction he developed infective sinusitis, which showed a poor response to broad spectrum antibiotics and antifungal agents. He rapidly deteriorated, developed a stroke, and died.

\section{Discussion}

Hairy cell leukaemia is characterised clinically by pancytopenia and splenomegaly. The disorder constitutes $2 \%$ of all leukaemias ${ }^{5}$ and runs a chronic course. Monocytopenia and granulocytopenia predispose the patients to opportunistic infections. ${ }^{6}$ The bone marrow usually reveals a dry tap as a result of fibrosis; however, bone marrow morphology is diagnostic. Hairy cells are large differentiated B cells with hair-like projections. They often express the $\mathrm{B}$ cell markers CD19, CD20, multiple immunoglobulin heavy chains, human major histocompatibility complex DR antigens, and CD45RA. They can also express monocytic markers CD11c and the interleukin 2 (IL-2) receptor CD25. ${ }^{5}$

The occurrence of second malignancies in HCL is well recognised. The second malignancy can occur at the same time or after the diagnosis of HCL. Jacobs et al reviewed 172 cases of HCL and $15(8.7 \%)$ were found to have second malignancies, two of which were haematological malignancies. There was no report of a myeloproliferative disorder in this series. $^{3}$ There are many other reported tumours of lymphoid organs associated with HCL, most commonly large cell lymphoma, but also myeloma and $\mathrm{T}$ cell leukaemia/ lymphoma. ${ }^{34}$
The association of myeloproliferative disorders with HCL is very rare. Mufti et al described one patient with HCL and polycythaemia rubra vera (PRV); however, in this patient, HCL occurred after the treatment of PRV with radioactive phosphorus. ${ }^{7}$ Kampmeier et al described one case of PRV that followed the treatment of HCL with interferon; however, no cytogenetic abnormalities were found. ${ }^{4}$ There is only one case of CML occurring in a patient with HCL reported in the literature. ${ }^{2}$ However, this case was atypical in a few ways, with an initial high white blood cell count, the absence of monocytopenia, and atypical morphology. Immunophenotyping also showed that hairy cells were CD25 negative. Our patient with HCL developed CML four years after remission. Cytogenetics confirmed the $t(9 ; 22)$ translocation. The patient ultimately developed ALL with additional chromosome abnormalities.

There are reports that prolonged interferon treatment leads to an increased incidence of second malignancies in HCL. Kampmeier et al reviewed 69 patients with HCL who were given interferon and found that six patients developed haematological malignancies. ${ }^{4}$ Three patients had malignant lymphoma, one patient developed acute myeloid leukaemia, and one developed PRV. However neither our patient nor that of Reeves et al received interferon. ${ }^{2}$ The study of Jacobs et al revealed two patients with HCL and haematological malignancies who had received chlorambucil. ${ }^{3}$ Our patient was treated with deoxycoformycin. Therefore, factors that lead to the development of second malignancies in HCL remain to be determined. It is possible that CML might have had its origin even before HCL was diagnosed in our patient; CML might have a prolonged silent or quiescent phase during which it can be cytogenetically detected despite an apparently normal blood count and film.

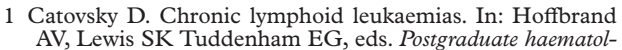
AV, Lewis SK Tuddenham EG, eds. Postgraduate haematology,

2 Reeves JE, Robbins BA, Pankey LR, et al. Simultaneous occurrence of variant hairy cell leukemia and chronic phase chronic myelogenous leukaemia. Cancer 1995;75:2089-92.

3 Jacobs RH, Vokes EE, Golombo HM. Second malignancies in hairy cell leukaemia. Cancer 1985;56:1462-67.

4 Kampmeier P, Spielberger R, Dickstein J, et al. Increased incidence of second neoplasms in patients treated with interferon alpha $2 \mathrm{~b}$ for HCL; a clinicopathologic assessment. Blood 1994;83:2931-8.

5 Haglund U, Juliusson G, Stellan B, et al. Hairy cell leukaemia is characterised by clonal chromosome abnormalities clustered to specific regions. Blood 1994;83:2637-45.

6 Golombo HM, Hanauer SB. Infectious complications assoGolombo HM, Hanauer SB. Infectious complication

7 Mufti GJ ,Hamblin TJ, Stevenson FK, et al. Polycythemia Mufti GJ,Hamblin TJ, Stevenson FK, et al. Polycythemia
rubra vera and hairy cell leukaemia in the same patient: studies on spleen. F Clin Pathol 1982;35:1312-15. 